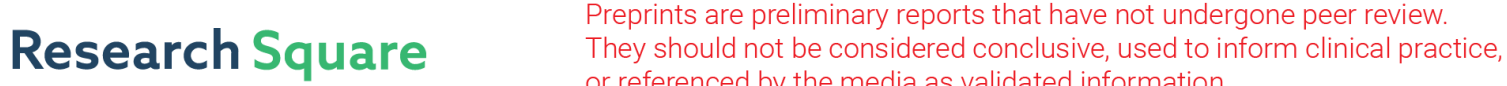 or referenced by the media as validated information. \\ Early and Late Complications After Abdominal Surgery in Patients With COVID-19 in Armenia
}

Karine Aloyan ( $\square$ aloyan_karine@yahoo.com )

Yerevan State Medical University

\section{Hayk Harutyunyan}

Yerevan State Medical University

Arayik Voskanyan

Yerevan State Medical University

\section{Research Article}

Keywords: COVID-19, Postoperative Complications, Surgical Mortality, Abdominal Surgery, Comorbidity

Posted Date: January 27th, 2021

DOI: https://doi.org/10.21203/rs.3.rs-136560/v1

License: (c) (1) This work is licensed under a Creative Commons Attribution 4.0 International License.

Read Full License 


\section{Early and Late Complications After Abdominal Surgery in Patients With COVID-19 in Armenia}

Karine Aloyan, MD; Hayk Harutyunyan, MD, PhD; Arayik Voskanyan, MD, PhD

Corresponding Authors: Karine Aloyan, MD, Department of Infectious Diseases, Yerevan State Medical University, Muratsan Hospital Complex, 114 Muratsan St, Yerevan 0074, Armenia (aloyan_karine@ yahoo.com); Hayk Harutyunyan, MD, PhD, Department of General Surgery, Yerevan State Medical University, Astghik Medical Center, 28 Daniel Varuzhani St, Yerevan 0032, Armenia (doctorhayk@yahoo.com).

Author Affiliations: Department of Infectious Diseases, Yerevan State Medical University, Muratsan Hospital Complex, Yerevan, Armenia (Aloyan); Department of General Surgery, Yerevan State Medical University, Astghik Medical Center, Yerevan, Armenia (Harutyunyan); Department of Endoscopic and Endocrine Surgery, Yerevan State Medical University, Astghik Medical Center, Yerevan, Armenia (Voskanyan). 


\section{Abstract}

Background Coronavirus disease 2019 (COVID-19) is one of the challenging topics in surgery nowadays. Available data indicate high surgical mortality and many postoperative complications in surgical patients with various stages of coronavirus infection. But still a little is known on early and late outcomes of abdominal surgery in patients with COVID-19, especially in countries with limited resources. In this article we describe early and late complications after abdominal surgery in patients recovering from COVID-19. We define early and late complications as those developing during 1-30 and 31-60 postoperative days, accordingly.

Methods A prospective cohort study is conducted at Astghik Medical Center, Yerevan, Armenia, from February 1 until October 31, 2020. The study population comprised 259 patients with COVID-19 and 245 patients without COVID-19 matched by operation type, age, sex, and comorbidities, underwent abdominal surgery. Differences between early (1-30 postoperative days) and late (31-60 postoperative days) complications in both groups were analyzed. Patients with COVID-19 had been diagnosed based on both clinical and laboratory (RT-PCR, reverse transcriptase-polymerase chain reaction assay in nasopharyngeal swabs) criteria at least 14 days before abdominal surgery. Patients without COVID-19 were not screened at time of surgery, but were free from any respiratory symptoms and had negative RT-PCR results during preoperative 14 days. The primary endpoints were early and late postoperative complications. Secondary endpoints were to determine 60-day surgical mortality and the impact of comorbidities as additional risk factor of postoperative complications in patients with COVID-19.

Results A total of 29 patients with COVID-19 developed early or late postoperative complications. Only 4 patients without COVID-19 with early postoperative complications were identified. The median age of patients with COVID-19 who had early and late postoperative complications were 54.5 (range: 45-64) and 69.5 (range: 65-74), correspondingly. At least one comorbidity was present in $25(86.2 \%)$ of 29 patients with COVID-19 who developed early or late postoperative complications. A 60 -day surgical mortality was $14.3 \%$.

Conclusion COVID-19 is associated with high risk for postoperative complications of abdominal surgery even if surgical procedures are performed after 14 days of COVID-19 onset. Only patients aged 45 to 74 years developed complications in our study. Presence of at least one comorbidity was an additional risk factor of postoperative complications. Larger and better designed studies are needed to find out indicators for early detection of postoperative complications in patients with COVID-19, especially in people older than 45 years and in those with comorbidities.

Keywords: COVID-19, Postoperative Complications, Surgical Mortality, Abdominal Surgery, Comorbidity. 


\section{Background}

Coronavirus disease 2019 (COVID-19) caused by the novel severe acute respiratory syndrome coronavirus 2 (SARS-CoV-2) has complex pathogenetic mechanisms affecting many organ systems, including gastrointestinal tract and liver. Reports published on the outcome of surgical procedures in COVID-19 patients indicate that COVID-19 is a highly challenging topic in surgery. Even though during the COVID-19 pandemic, hospitals recommend postponing surgeries, if possible ${ }^{1}$, many patients still need emergent, urgent, or semi-urgent procedures. Studies revealed high mortality of surgical patients with active and asymptomatic coronavirus infection varying from $14.3-38.5 \%$ and depending on whether the surgery performed electively or urgently $y^{2-10}$. Poor surgical outcomes defined as prolonged hospital stay, perioperative complications and high mortality, were most frequent in general surgery or oncological surgery patients, in individuals 65 year and older and in those with chronic comorbidities ${ }^{8,11}$.

We conducted a cohort study to investigate early and late complications after abdominal surgery in patients with COVID-19 in Armenia. Taking into account prolonged course of coronavirus infection, we selected patients in their clinical recovery period, ie after 14 days of COVID-19 onset and looked for complications during 60 postsurgical days, that is a longer period than usually used in surgical practice.

\section{Methods}

We conducted a prospective cohort study to investigate complications in patients with COVID19 after abdominal surgery performed at Astghik Medical Center of Yerevan, Armenia between February 1 and August 31, 2020. A total of 259 patients in clinical recovery period of COVID-19 (>14 days after disease onset) were selected as cases. All of them had been diagnosed with COVID-19 by clinical and laboratory criteria (RT-PCR assay in nasopharyngeal swabs). All of them had moderate forms of COVID-19 with lung affection of various degree on time of COVID-19 diagnosis and oxygen saturation $\geq 94 \%$ at the time of the surgery. Patients with mild or severe forms of COVID-19, those admitted within first 14 days of COVID-19 or being diagnosed based on only one criterion (either clinical, or laboratory) have been excluded from the study. The 245 controls were selected and matched for age, sex, comorbidities, and surgery type and urgency to the cases. No screening RT-PCR was performed at the hospital before surgery due to limited resources, however, they had documented outpatient data of negative RTPCR results within preoperative 14 days and were screened for absence of any recent (14-day) close contact with COVID-19 patients. Patients from both groups were taken under dynamic medical control for 60 days (until October 31, 2020). All patients gave oral consent to use their medical information for the study. Differences in postoperative complications between the two groups were analyzed. We defined early and late postoperative complications as those developing during 1-30 and 31-60 postoperative days, respectively. The presence of at least one comorbidity as an additional risk factor for postoperative complications and 60-day surgical mortality was observed as secondary endpoints of the study. 
Study demographics and characteristics of patients with or without COVID-19 are presented in Table 1.

Table 1. Demographics and Characteristics of Surgical Patients With or Without COVID-19

\begin{tabular}{|c|c|c|}
\hline & $\begin{array}{l}\text { Patients with } \\
\text { COVID-19 }\end{array}$ & $\begin{array}{l}\text { Patients without } \\
\text { COVID-19 }\end{array}$ \\
\hline No. of patients & 259 & 245 \\
\hline \multicolumn{3}{|l|}{ Sex, No. $(\%)$} \\
\hline Male & $121(46.7)$ & $110(44.9)$ \\
\hline Female & $138(53.3)$ & $135(55.1)$ \\
\hline \multicolumn{3}{|c|}{ Age groups, median (range), y, No. $(\%)$} \\
\hline $31.5(19-44)$ & $46(17.7)$ & $40(16.3)$ \\
\hline $54.5(45-64)$ & $88(34)$ & $82(33.5)$ \\
\hline $69.5(65-74)$ & $125(48.3)$ & $123(50.2)$ \\
\hline Presence of $\geq 1$ comorbidity, No. $(\%)$ & $98(37.8)$ & $102(41.6)$ \\
\hline \multicolumn{3}{|l|}{ Comorbidity, No $(\%)$} \\
\hline Diabetes $^{\mathrm{a}}$ & $33(12.7)$ & $30(12.2)$ \\
\hline Cardiovascular disease $^{\mathrm{b}}$ & $28(10.8)$ & $32(13.1)$ \\
\hline COPD & $20(7.7)$ & $16(6.5)$ \\
\hline Chronic kidney disease & $11(4.2)$ & $8(3.3)$ \\
\hline Other & $14^{\mathrm{c}}(5.4)$ & $17^{\mathrm{d}}(6.9)$ \\
\hline \multicolumn{3}{|c|}{$\begin{array}{l}\text { a Type } 2 \text { diabetes with metabolic syndrome or obesity (BMI > 30) } \\
{ }^{b} \text { Hypertension or ischemic heart disease } \\
{ }^{c} \text { Autoimmune hepatitis, deep venous thrombosis, hyperthyroidism, or combination of } 2 \\
\text { comorbidities } \\
{ }^{\mathrm{d}} \text { Chronic hepatitis C, rheumatoid arthritis, osteoarthritis, or combination of } 2 \text { comorbidities } \\
\text { Abbreviation: BMI, body mass index; COPD, chronic obstructive pulmonary disease }\end{array}$} \\
\hline
\end{tabular}




\section{Results}

Thirty-six patients with COVID-19 died in early postoperative period. The median age of them was 63.5 (range: 52-74). Males and females were 52\% and 48\%, respectively. All of them had at least one comorbidity. No patient without COVID-19 died during the study.

Twenty-nine (11.2\%) patients with COVID-19 and four (1.6\%) patients without COVID-19 developed postoperative complications. Early and late postoperative complications in patients with COVID-19 were 21 and 8, accordingly. All four complications described in patients without COVID-19 were early.

Patients with COVID-19 who developed early postoperative complications had median age of 54.5 (range: 45-64). Males and females were 11 and 10, respectively. At least one comorbidity was present in 17 of $21(80.9 \%)$ patients. The spectrum of early complications was paralytic ileus, small bowel ischemia, pancreatitis, and moderate (5 to 10 times the upper reference limit) transaminitis. All 21 patients recovered with therapeutic treatment only.

The median age of the patients with late postoperative complications was 69.5 (range: 65-74). The numbers of male and female patients were equal. All of them had at least one comorbidity. The spectrum of late complications consisted of spleen infarction, abdominal abscess, necrotizing pancreatitis, total intestinal necrosis, and pleural empyema. Three patients required minimal invasive procedures. Five patients underwent major surgery, one of them died due to total intestinal necrosis.

The characteristics of early and late postoperative complications and their outcomes are presented in Table 2.

In patients without COVID-19 we observed only early postoperative complications. All of them were 65 years or older (median age 69.5 within 65-74 range) with one or more comorbidities. Three of them were male, one patient was female. We revealed paralytic ileus in two patients, surgical wound infection in one patient, and moderate transaminitis in one patient. All of them recovered with therapeutic treatment.

During study 37 patients with COVID-19 died, thus 60-day surgical mortality was $14.3 \%$. 
Table 2. Characteristics and Outcomes of Early and Late Complications in Patients With COVID-19 After Abdominal Surgery

\begin{tabular}{|c|c|c|}
\hline & \multicolumn{2}{|c|}{ Postoperative complications } \\
\hline & Early (1-30 postoperative days) & Late (31-60 postoperative days) \\
\hline No. of patients & 21 & 8 \\
\hline Age median, y (range) & $54.5(45-64)$ & $69.5(65-74)$ \\
\hline Sex, No. $(\%)$ & & \\
\hline Male & $11(52.4)$ & $4(50)$ \\
\hline Female & $10(47.6)$ & $4(50)$ \\
\hline $\begin{array}{l}\text { Presence of } \geq 1 \text { comorbidity } \\
\text { No. of patients }(\%)\end{array}$ & $17(80.9)$ & $8(100)$ \\
\hline $\begin{array}{l}\text { Spectrum of complications } \\
\text { No. of patients }\end{array}$ & $\begin{array}{l}\text { Paralytic ileus, } n=13 \\
\text { Small bowel ischemia, } n=4 \\
\text { Pancreatitis, } n=2 \\
\text { Transaminitis, } n=2\end{array}$ & $\begin{array}{l}\text { Spleen infarction, } n=3 \\
\text { Abdominal abscess, } n=2 \\
\text { Necrotizing pancreatitis, } n=1 \\
\text { Small bowel ischemia with total } \\
\text { necrosis, } n=1 \\
\text { Pleural empyema, } n=1\end{array}$ \\
\hline Outcomes, No. (\%) & $\begin{array}{l}\text { Recovery with therapeutic } \\
\text { treatment, } n=21(100)\end{array}$ & 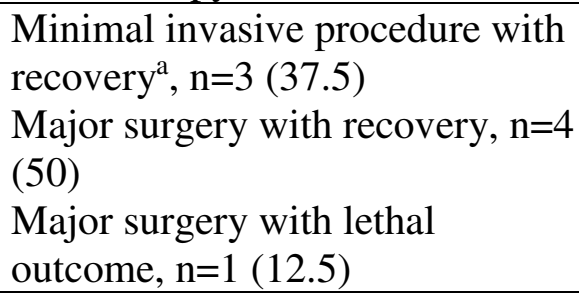 \\
\hline
\end{tabular}

\section{Discussion}

In our study significant association between COVID-19 and postoperative complications, as well as, high surgical mortality was found. Even having the operative procedures performed after 14 days of the disease onset, COVID-19 is responsible for high incidence of postoperative complications (11.2\% vs $1.6 \%$ in patients without COVID-19). Early postoperative complications were more prevalent than late ones $(72.4 \%$ vs $27.6 \%)$ in patients with COVID-19, however late complications tended to be more severe. People aged 65 and older developed mostly late complications. These patients had nonspecific symptoms for a long time, such as low-grade fever or short spikes of high-grade fever, malaise, diffuse abdominal pains, however, they considered the symptoms as manifestations of the post coronavirus syndrome and sought medical help late. Many possible explanations starting from decreased immunity, more prolonged consequences of COVID-19 in older adults and finishing with socioeconomic factors and low literacy can be supposed.

The presence of at least one comorbidity was noticed as an additional risk factor for developing postoperative complications. Twenty-five (86.2\%) patients with COVID-19 from those who 
develop early or late postoperative complications had at least one comorbidity, more commonly, severe hypertension, poorly controlled type 2 diabetes (fasting blood glucose $>130 \mathrm{mg} / \mathrm{dL}$ (7.2 $\mathrm{mmol} / \mathrm{L}$ ) within preoperative 3 months) with metabolic syndrome or obesity (BMI > 30), prolonged ischemic heart disease (more than 5 years), and hypertension managing with two or more medications.

The pathogenetic mechanisms described in COVID-19 such as binding angiotensin-converting enzyme 2 (ACE2) present in many cell types and tissues ${ }^{2,12-15}$, including digestive system, as well as, promoting hypercoagulable states mostly illicit the spectrum of postoperative complications that we diagnosed during our study, such as small bowel ischemia with or without necrosis, spleen infarction, transaminitis, pancreatitis with or without necrosis. In many patients with COVID-19 who died or developed postoperative complications we detected the preoperative laboratory abnormalities indicating the involvement of gastrointestinal system, such as elevated levels of transaminases, hypoproteinemia, prothrombin time prolongation, along with high levels of fibrinogen in blood and lymphopenia. However, further investigation of these data was not performed as we could not match them with patients without COVID-19 due to limited resources.

As reported previously ${ }^{2,16-19}$, severe cases of COVID-19 usually present in the older adults and in those with an underlying disease, remarkably, hypertension, cardiovascular disease, respiratory system disease, diabetes, obesity, chronic kidney disease, and immunocompromised states. Our patients with COVID-19 who developed poor surgical outcomes (death or postoperative complications) had median age of 59.5 year (range: $45-74)$; most of them (93.4\%) had one or more comorbidities. Therefore, increasing age and presence of at least one comorbidity are additional risk factors for postoperative poor outcomes; nevertheless, strict matching without potential confounders is required.

Based on literature data patients with COVID-19 who underwent surgical procedures develop serious perioperative complications such as thromboembolic disease, pulmonary complications, thrombocytopenia, secondary bacterial infections, cardiac and renal failure ${ }^{1,2,6-9}$. High mortality of surgical patients with active and asymptomatic coronavirus infection varying from 14.3 $38.5 \%$ is documented ${ }^{1-10,20}$. In our study 60-day surgical mortality in patients with COVID-19 was $14.3 \%$. Despite being unacceptably high, it is still one of the lowest described in the literature, that can be judged as a positive result for the country with limited resources. The reasons of death in 36 patients who died in early postoperative period were not included as early postoperative complications because of inability to perform postmortem analysis in patients with COVID-19 in Armenia and exclude comorbidities as confounders.

More frequent postoperative follow-up checks of surgical patients with COVID-19 were recommended by authors ${ }^{21,22}$ without precision of duration. Taking into account the data derived from our study, we recommend postsurgical follow-up at least for 60 days, especially in older adults and in patients with comorbidities. Any nonspecific symptom treated as a sign for a postoperative complication can decrease the chance of lethal outcome as it was in our patient with total intestinal necrosis who died vs them with small bowel ischemia diagnosed and treated earlier. 
Limitations Several limitations to the study include limited resources and inability to match patients based on laboratory abnormalities; absence of postmortem analysis; small study size with noncomparable outcomes and inability to eliminate confounders, such as age, sex, and comorbidities. Nonetheless, our study described the risk of early and late complications after abdominal surgery in patients with COVID-19 along with the impact of increasing age and comorbidities in both with or without COVID-19 groups. These findings warrant further studies with larger sample size and more parameters of matching, including but not limited to laboratory abnormalities that are characteristic for coronavirus infection.

\section{Conclusions}

COVID-19 is an independent risk factor for both early and late postoperative complications after abdominal surgery and high surgical mortality. Patients aged 45 to 74 years and those with one or more comorbidities have higher risk of postoperative complications. Better designed studies are required to detect all risk factors, especially manageable ones, thus to find out preventive strategies for poor surgical outcomes.

\section{Abbreviations}

COVID-19, coronovirus disease 2019

RT-PCR, reverse transcriptase-polymerase chain reaction

SARS-CoV-2, severe acute respiratory syndrome coronavirus 2

BMI, body mass index

COPD, chronic obstructive pulmonary disease

\section{Acknowledgements}

Author Contributions: Drs Aloyan and Harutyunyan had full access to all data in the study and take responsibility for the integrity of the data and the accuracy of the data analysis.

Concept and design: Aloyan.

Acquisition, analysis, or interpretation of data: All authors.

Drafting of the manuscript: All authors.

Critical revision of the manuscript for important intellectual content: Aloyan.

Statistical analysis: Aloyan.

Administrative, technical, or material support: Harutyunyan, Voskanyan.

Supervision: Aloyan.

Conflict of Interest Disclosures: No conflict of interest to report. 


\section{References}

1. Doglietto F, Vezzoli M, Gheza F, Lussardi GL, Domenicucci M, Vecchiarelli L, et al. Factors associated with surgical mortality and complications among patients with and without coronavirus disease 2019 (COVID-19) in Italy. JAMA Surg. 2020;155(8):114. doi.org/10.1001/jamasurg.2020.2713.

2. Abate SM, Mantefardo B, \& Basu B. Postoperative mortality among surgical patients with COVID-19: a systematic review and meta-analysis. Patient Safety in Surgery. 2020;14(1):37. doi.org/10.1186/s13037-020-00262-6.

3. Seeliger B, Philouze G, Cherkaoui Z, Felli E, Mutter D, Pessaux P. Acute abdomen in patients with SARS-CoV-2 infection or co-infection. Langenbeck's Arch Surg. 2020;405:861-866. doi: 10.1007/s00423-020-01948-2.

4. Cai Y, Hao Z, Gao Y, Ping W, Wang Q, Peng S, et al. COVID-19 in the perioperative period of lung resection: a brief report from a single thoracic surgery department in Wuhan. China. J Thorac Oncol. 2020;15(6):10651072. doi.org/10.1016/j.jtho.2020.04.003.

5. Peng S, Huang L, Zhao B, Zhou S, Braithwaite I, Zhang N, et al. Clinical course of coronavirus disease 2019 in 11 patients after thoracic surgery and challenges in diagnosis. J Thorac Cardiovasc Surg. 2020;160(2):585-

593. doi.org/10.1016/j.jtcvs.2020.04.005.

6. Egol KA, Konda SR, Bird ML, Dedhia N, Landes EK, Ranson RA, et al. Increased mortality and major complications in hip fracture care during the COVID-19 pandemic: a New York City perspective. J Orthop Trauma. 2020;34(8):395340. doi.org/10.1097/bot.0000000000001845.

7. Lei S, Jiang F, Su W, Chen C, Chen J, Mei W, et al. Clinical characteristics and outcomes of patients undergoing surgeries during the incubation period of COVID-19 infection. Eclinical Medicine. 2020;21:1-8. doi.org/10.1016/j.eclinm.2020.100331.

8. Kayani B, Onochie E, Patil V, Begum F, Cuthbert R, Ferguson D, et al. The effects of COVID-19 on perioperative morbidity and mortality in patients with hip fractures. Bone Joint J. 2020;102-B(9):1136-1145. doi: 10.1302/0301-620X.102B9.BJJ-2020-1127.R1.

9. Nahshon C, Bitterman A, Haddad R, Hazzan D, Lavie O. Hazardous postoperative outcomes of unexpected COVID-19 infected patients: a call for global consideration of sampling all asymptomatic patients before surgical treatment. World J Surg. 2020;44(8):2477-2481. doi: 10.1007/s00268-020-05574-2.

10. Thyagarajan R, Mondy K. Timing of surgery after recovery from coronavirus disease 2019 (COVID-19) infection. Infect Control Hosp Epidemiol. 2020;7(3):1-2. doi: 10.1017/ice.2020.325. 
11. Aziz H, Filkins A, Kwon YK. Review of COVID-19 outcomes in surgical patients. Am Surg. 2020;86(7):741-745. doi.org/10.1177\%2F0003134820934395.

12. Radzikowska U, Ding M, Tan G, Zhakparov D, Peng Y, Wawrzyniak P, et al. Distribution of ACE2, CD147, CD26, and other SARS-CoV-2 associated molecules in tissues and immune cells in health and in asthma, COPD, obesity, hypertension, and COVID-19 risk factors. Allergy. 2020;75(11):2829-2845. doi: 10.1111/all.14429.

13. Du F, Liu B, Zhang S. COVID-19: the role of excessive cytokine release and potential ACE2 down-regulation in promoting hypercoagulable state associated with severe illness. J Thromb Thrombolysis. 2020;16:1-17. doi: 10.1007/s11239-020-02224-2.

14. Hajifathalian K, Krisko T, Mehta A, Kumar S, Schwartz R, Fortune B, et al; WCM-GI research group. Gastrointestinal and hepatic manifestations of 2019 novel coronavirus disease in a large cohort of infected patients from New York: clinical implications. Gastroenterology. 2020;159(3):1137-1140.e2. doi.org/10.1053/j.gastro.2020.05.010.

15. Kumar A, Arora A, Sharma P, Anikhindi SA, Bansal N, Singla V, et al. Gastrointestinal and hepatic manifestations of Corona virus Disease-19 and their relationship to severe clinical course: a systematic review and meta-analysis. Indian J Gastroenterol. 2020;39:268-284. doi: 10.1007/s12664-020-01058-3.

16. Yang J, Zheng Y, Gou X, Pu K, Chen Z, Guo Q, et al. Prevalence of comorbidities in the novel Wuhan coronavirus (COVID-19) infection: a systematic review and meta-analysis. Int J Infect Dis. 2020;94:91-95. doi: 10.1016/j.ijid.2020.03.017.

17. Emami A, Javanmardi F, Pirbonyeh N, Akbari A. Prevalence of underlying diseases in hospitalized patients with COVID-19: a systematic review and meta-analysis. Arch Acad Emerg Med. 2020;8:e35.

18. Guan WJ, Liang WH, Zhao L, Liang HR, Chen ZS, Li YM, et al. Comorbidity and its impact on 1590 patients with Covid-19 in China: a nationwide analysis. Eur Respir J. 2020;55:2000547. doi.org/10.1183/13993003.00547-2020.

19. Richardson S, Hirsch JS, Narasimhan M, Crawford JM, McGinn T, Davidson KW, et al. Presenting characteristics, comorbidities, and outcomes among 5700 patients hospitalized with COVID-19 in the New York City area. JAMA. 2020;323(20):20522059. https://doi.org/10.1001/jama.2020.6774.

20. Moheb ME, Naar L, Christensen MA, Kapoen AC, Maurer LR, Farhat M, et al. Gastrointestinal complications in critically ill patients with and without COVID-19. JAMA. 2020;324(18):1899-1901. doi:10.1001/jama.2020.19400.

21. Gao Y, Xi H, Chen L. Emergency surgery in suspected COVID-19 patients with acute abdomen: case series and perspectives. Ann Surg. 2020;272(1). doi.org/10.1097/SLA.0000000000003961. 
22. Zhou F, Yu T, Du R, Fan G, Liu Y, Liu Z, et al. Clinical course and risk factors for mortality of adult inpatients with COVID-19 in Wuhan, China: a retrospective cohort study. Lancet 2020; 395:1054-1062. doi.org/10.1016/S0140-6736(20)30566-3. 